\title{
Danger and usefulness: An alternative framework for understanding rapid evaluation effects in perception?
}

\author{
LEE H. WURM \\ Wayne State University, Detroit, Michigan
}

\begin{abstract}
Previous studies have shown effects of rated danger and usefulness on lexical access. All of them have used stimuli selected for connotations of danger and/or usefulness. Stimuli for the present lexical decision study were all of the nouns, verbs, and adjectives from the Balota et al. (2002) English Lexicon Project (subject to constraints relating to experimental control; none had anything to do with danger or usefulness). The interaction between danger and usefulness ratings previously demonstrated (Wurm \& Vakoch, 2000; Wurm, Vakoch, Seaman, \& Buchanan, 2004; Wurm, Whitman, Seaman, Hill, \& Ulstad, 2007) was found for nouns, even when age of acquisition was controlled. It was also found for verbs and adjectives. The interaction is believed to reflect competing pressures to (1) avoid dangerous objects/events and (2) approach valuable resources. It may be a manifestation of the rapid evaluation effects pervasive in the literature. Post hoc analyses showed that danger and usefulness explain as much variance as valence and arousal, or evaluation, potency, and activity.
\end{abstract}

Research demonstrates pervasive effects of certain connotative dimensions on cognitive/perceptual performance. The most commonly discussed dimension is a good/bad dimension called evaluation or valence. It has anchored several theoretical accounts, usually in combination with other dimensions such as potency (strong/weak), activity (fast/slow), or arousal (e.g., Osgood, 1969; Robinson, Storbeck, Meier, \& Kirkeby, 2004). Effects of these variables have even been observed in lexical decision and naming (Wurm, Labouvie-Vief, Aycock, Rebucal, \& Koch, 2004; Wurm, Vakoch, \& Seaman, 2004). This is surprising, because these are conceptualized as low-level perceptual tasks that should not show influences of semantic variables.

Wurm and Vakoch (2000) noted the interpretational ambiguity of a framework built around evaluation or valence: What does good mean? Good for whom, and to what end? Theorists long ago related valence to approach and avoidance behaviors, and to adaptiveness for survival (e.g., Lewin, 1935; Schneirla, 1959), ${ }^{1}$ but how are we to think about items with good and bad connotations (e.g., electricity, elephant, pesticide, syringe)? We attempted to address the issue by measuring the constructs of danger and usefulness (Wurm \& Vakoch, 2000). Why do we avoid things? One answer is because they are dangerous. Why do we approach things? Because they are useful in some way.

In a series of studies, we found that words with higher usefulness or danger ratings are recognized faster and more accurately, controlling for several variables (Wurm
\& Vakoch, 2000; Wurm, Vakoch, Aycock, \& Childers, 2003; Wurm, Vakoch, Seaman, \& Buchanan, 2004; Wurm, Whitman, Seaman, Hill, \& Ulstad, 2007). These studies used auditory lexical decision, visual lexical decision, and auditory naming, so the effects generalize across tasks and modalities. The usefulness effect is robust, as is the danger $\times$ usefulness interaction: Increasing danger predicts faster RTs for words rated low on usefulness, but slower RTs for words rated high on usefulness. Our interpretation is that items high on usefulness and danger engage both approach and avoid response tendencies; the slowed RTs in this context reflect a response conflict.

Although we demonstrated the effects with three different tasks, it is necessary to establish generalizability for several reasons. First, all the studies used the same 100 common nouns (or a subset of them). These stimuli were chosen to fill a $2 \times 2$ semantic space defined by high and low danger and usefulness. The generalizability of the results across words therefore remains unknown. It is crucial to understand whether danger and usefulness effects are as pervasive as evaluation effects seem to be, because I will speculate that danger and usefulness provide an alternative framework in which evaluation effects can be understood.

In addition, previous studies did not control for age of acquisition (AoA), which has been shown to affect lexical retrieval (Ellis \& Morrison, 1998) and is hypothesized to be relevant in object name learning (Zevin \& Seidenberg, 2002). It is possible that danger and usefulness effects are confounded with this variable. 
Finally, it is unknown whether the effects apply to parts of speech other than nouns. No models of word recognition can accommodate effects of danger and usefulness (or evaluation/valence, or any other such dimensions). Thus, this is largely unexplored territory, but it is nevertheless important to determine the scope of the effects.

\section{Stimulus Selection}

From the English Lexicon Project (Balota et al., 2002), I selected all items that are monomorphemic, are not homophones or homographs, are listed as only one part of speech with nonzero frequency in the CELEX lexical database (Baayen, Piepenbrock, \& Gullikers, 1995), and have a value listed in the MRC psycholinguistic database (Michael Wilson, 1988) on meaningfulness, concreteness, familiarity, and imageability. In addition, if the word was a noun, it had to have an AoA value in the MRC database (very few nonnouns have an AoA value). ${ }^{2}$ As a result, 175 words were used as stimuli: 104 nouns, 34 verbs, and 37 adjectives.

\section{PRELIMINARY RATING STUDY}

\section{Method}

Participants. A total of 144 native speakers of English participated for course credit. Half rated each word on danger and half rated each word on usefulness.

Materials. The stimuli were described above.

Procedure. Using a keyboard, participants rated each word on its danger or usefulness. A rating scale appeared onscreen, with endpoints 1 and 8 labeled Not at all useful for human survival and Extremely useful for human survival, respectively. Ratings for danger worked the same way, with the word "dangerous" substituted for "useful."

\section{Results and Discussion}

For each item, the mean danger rating and mean usefulness rating were calculated and used as predictors in the main experiment. Mean danger ratings ranged from 1.31 to $6.33(M=3.82, S D=3.56)$. Mean usefulness ratings ranged from 2.28 to $7.40(M=4.84, S D=3.62)$. Danger and usefulness ratings were not correlated $[r(173)=$ $.069, p=.36]$. Appendix A lists the mean ratings for each stimulus.

\section{EXPERIMENT Auditory Lexical Decision}

\footnotetext{
Method

Participants. Fifty-eight native speakers of English participated for course credit. None participated in the rating study.

Materials. The stimuli described above were used. To create an equal number of pseudowords, I randomly chose a word from CELEX and changed one phoneme at random to another phoneme from the same class.

The stimuli were spoken by a native speaker of English who was unfamiliar with the purpose or hypotheses of the study. Words ranged in duration from 301 to $738 \mathrm{msec}(M=506, S D=82)$. Pseudowords ranged in duration from 233 to $818 \mathrm{msec}(M=513$, $S D=93)$. The word/pseudoword difference in mean duration did not approach significance $(p=.46)$.

Procedure. Stimuli were played over headphones in a single block, with an ISI of 1,500 msec. Participants made speeded lexical
}

decisions by pressing the right button on a two-button mouse for words and the left button for pseudowords. (The mouse was placed directly in front of the participant - no keyboard was present.) Each stimulus was heard once by each participant. A different random order was used for each participant. RTs were measured from the acoustic onset of each stimulus.

Data analysis. RTs were analyzed with a multilevel linear mixedeffects ANCOVA with $\log$ RT as the dependent variable and participant as random effect (Pinheiro \& Bates, 2000). Appendix B lists variables included for purposes of control or ruling out alternative explanations. Several variables were transformed in order to remove skewness in their distributions and minimize the effect of atypical outliers: word frequency, number of competitors, number of meanings, concreteness, morphological family size, summed bigram frequency, danger, and usefulness. The danger and usefulness variables were centered, to reduce collinearity and aid interpretation of the interaction coefficients.

\section{Results and Discussion}

RTs were discarded if they were faster than $300 \mathrm{msec}$ ( 2 of the 10,150 RTs on real-word trials; $0.02 \%$ of the data) or slower than 2,000 msec from item onset ( $84 \mathrm{RTs} ; 0.83 \%$ ). RTs for incorrect trials were not analyzed (8.87\%).

Table 1 shows the results of the analysis. RTs were slower if an error occurred on the previous trial, indicating a realerting effect. Words with voiced onsets were recognized faster than those with unvoiced onsets, as Baayen, Wurm, and Aycock (in press) also found. Words higher in frequency, concreteness, familiarity, or morphological family size had faster RTs. Later uniqueness point (UP) locations, longer durations, higher written-to-spoken frequency ratios (indicating a later age of acquisition), or denser neighborhoods (as measured either by Coltheart's $N$ or the auditory competitor measure) were associated with slower RTs. Danger and usefulness both had facilitative main effects. All of these effects are in the expected directions.

Figure 1 shows the partial effect of each significant continuous predictor when the other predictors are held constant at their medians. Because the $y$-axes use the same range of values for each predictor, the figure provides immediate insight into the relative effect sizes of the predictors.

The crucial danger $\times$ usefulness interaction was significant $(p<.01)$. Figure 2 plots the regression equation with all variables except danger and usefulness held constant at their medians. For plotting purposes, danger was a vector running from the minimum to the maximum (transformed) danger value in the item set. Values one $S D$ below and above the median were used for "Low" and "High" usefulness. The slope relating danger to RT becomes more inhibitory as usefulness rises. This is precisely the pattern found in previous studies with common nouns, interpreted as reflecting the competing pressures to avoid danger and approach valuable resources.

The three-way interaction between danger, usefulness, and part of speech was also significant. Contrast coefficients (see Table 1) indicated that the interaction did not differ for verbs or for nouns, when compared with the reference case (adjectives). A follow-up analysis revealed that the interaction was significantly stronger for verbs than for nouns $[t(7273)=2.3675, p<.05]$. However, 
Table 1

Summary of the Multilevel ANCOVA for Variables Predicting Log Auditory Lexical Decision Time

\begin{tabular}{|c|c|c|c|c|c|}
\hline Variable & $\begin{array}{l}\text { Regression } \\
\text { Coefficient } \\
\quad(B)\end{array}$ & $\begin{array}{l}\text { Standard } \\
\text { Error } \\
\text { of } B\end{array}$ & $d f$ & $t$ & $p$ \\
\hline \multicolumn{6}{|l|}{ Main effects } \\
\hline $\operatorname{POS}(\mathrm{N})^{\mathrm{a}}$ & 0.0016 & 0.0079 & 9050 & 0.2000 & .8415 \\
\hline $\operatorname{POS}(\mathrm{V})^{\mathrm{a}}$ & 0.0046 & 0.0070 & 9050 & 0.6521 & .5143 \\
\hline Previous trial incorrect & 0.0305 & 0.0071 & 9050 & 4.3239 & .0000 \\
\hline Voiced onset phoneme & -0.0252 & 0.0043 & 9050 & -5.8563 & .0000 \\
\hline Stressed first syllable & 0.0083 & 0.0070 & 9050 & 1.1767 & .2394 \\
\hline Trial number & 0.0000 & 0.0000 & 9050 & -1.1060 & .2687 \\
\hline Word frequency & -0.0122 & 0.0017 & 9050 & -9.0730 & .0000 \\
\hline Uniqueness point location & 0.0003 & 0.0000 & 9050 & 9.2946 & .0000 \\
\hline Duration & 0.0003 & 0.0000 & 9050 & 7.0672 & .0000 \\
\hline Concreteness & -0.0020 & 0.0006 & 9050 & -3.2866 & .0010 \\
\hline Familiarity & -0.0005 & 0.0001 & 9050 & -7.6249 & .0000 \\
\hline Meaningfulness & 0.0000 & 0.0000 & 9050 & -1.0479 & .2947 \\
\hline Written-to-spoken ratio & 0.0147 & 0.0020 & 9050 & 7.5145 & .0000 \\
\hline Morphological family size & -0.0121 & 0.0030 & 9050 & -3.9760 & .0001 \\
\hline Number of meanings & 0.0063 & 0.0035 & 9050 & 1.8042 & .0712 \\
\hline Coltheart's $N$ & 0.0228 & 0.0030 & 9050 & 7.6721 & .0000 \\
\hline Summed bigram frequency & 0.0046 & 0.0036 & 9050 & 1.2807 & .2003 \\
\hline Number of competitors & 0.0086 & 0.0018 & 9050 & 4.8896 & .0000 \\
\hline Danger & -0.0131 & 0.0057 & 9050 & -2.3147 & .0207 \\
\hline Usefulness & -0.0455 & 0.0107 & 9050 & -4.2611 & .0000 \\
\hline \multicolumn{6}{|l|}{ Interactions } \\
\hline Danger $\times \operatorname{POS}(N)^{a, b}$ & -0.0137 & 0.0137 & 9045 & -1.0001 & .3173 \\
\hline Danger $\times \operatorname{POS}(\mathrm{V})^{\mathrm{a}, \mathrm{b}}$ & 0.0261 & 0.0188 & 9045 & 1.3830 & .1667 \\
\hline Usefulness $\times$ POS $(N)^{a, c}$ & -0.0118 & 0.0244 & 9045 & -0.4838 & .6285 \\
\hline Usefulness $\times$ POS $(\mathrm{V})^{\mathrm{a}, \mathrm{c}}$ & 0.0066 & 0.0301 & 9045 & 0.2206 & .8254 \\
\hline Danger $\times$ usefulness & 0.0679 & 0.0222 & 9045 & 3.0566 & .0022 \\
\hline Danger $\times$ usefulness $\times$ POS $(N)^{\mathrm{a}, \mathrm{d}}$ & -0.1116 & 0.0682 & 9043 & -1.6373 & .1016 \\
\hline Danger $\times$ usefulness $\times$ POS $(V)^{\mathrm{a}, \mathrm{d}}$ & 0.2439 & 0.1429 & 9043 & 1.7067 & .0879 \\
\hline
\end{tabular}

aPOS $=$ part of speech. Adjective is the reference group (determined by the software based on alphabetical order) and is mapped to the intercept of the model. b ${ }^{b}$ For danger $\times \operatorname{POS}[F(2,9045)=$ $2.95, p=.0526] . \quad{ }^{c}$ For usefulness $\times \operatorname{POS}[F(2,9045)=0.40, p=.6729] . \quad$ dFor danger $\times$ usefulness $\times$ POS $[F(2,9043)=4.91, p=.0074]$.

the danger $\times$ usefulness interaction was significant for all three parts of speech, as revealed by planned comparisons [nouns, $B=0.0590, S E B=0.0253, t(5427)=$ $2.3335, p=.0197 ;^{3}$ verbs, $B=0.6971, S E B=0.1441$, $t(1773)=4.8386, p<.0001$; adjectives, $B=0.2822, S E$ $B=0.0881, t(1697)=3.2019, p=.0014]$.

To ensure that the results were not due to statistical overfitting, I ran a second analysis retaining only word frequency, duration, and UP location as control variables. The danger $\times$ usefulness interaction remained significant $(p<.001$; see Figure 3$)$.

\section{GENERAL DISCUSSION}

This study clearly demonstrates that danger and usefulness effects are not restricted to particular stimuli or to one part of speech. The similarity to our previous studies is striking: We have always found a significant coefficient for usefulness, 2-6 times larger than that for danger. In the present study, the usefulness coefficient was 3.5 times larger than the danger coefficient, and the interaction was significant, as it has been in four of our five previous experiments. I believe that this interaction reflects the competing pressures between approach and avoid response tendencies: We must avoid danger, but approach useful things.

The effects generalize not only across tasks and modalities (Wurm et al., 2003; Wurm, Vakoch, Seaman, \& Buchanan, 2004), but also across a large sample of items chosen without respect to their connotations of danger or usefulness. The results are not due to uncontrolled effects of AoA, concreteness, familiarity, or any other of a large number of variables (see Appendix B). That the interaction holds for verbs might have been expected, given their nature. Finding the same interaction for adjectives, most of which seem nearly devoid of behavioral connotations, illustrates the truly pervasive nature of the effect. All items for which the control variables are available were included in the present study.

It is unorthodox to suggest that such effects would be present in the relatively abstract perceptual task of word recognition, and current models cannot accommodate these findings. Why should words, which are arbitrary mediators between objects/events and perceivers, elicit approach and avoidance movements? Preverbal infants demonstrate appropriate approach and avoidance movements to objects/events, and it is possible that as words are learned to refer to these objects/events, the danger and 

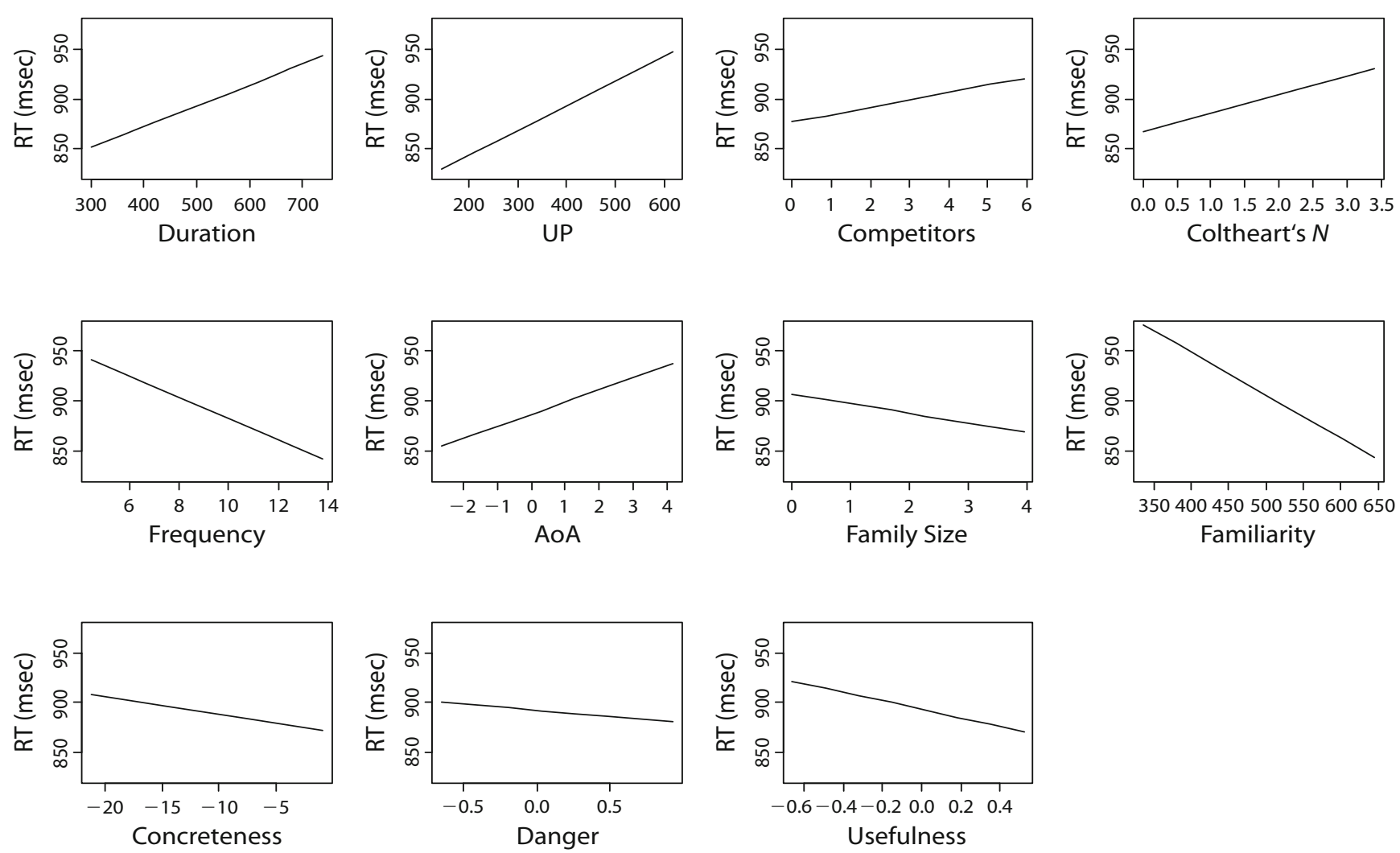

Figure 1. Partial-effects plot. Significant effects are shown, all on the same scale of $y$-axis values. RTs were log-transformed for the analysis, but untransformed RTs are shown.

usefulness associated with the thing being signified also come to be associated with the sign (e.g., Solarz, 1960).

Danger and usefulness may thus become part of the total meaning of a word. Wurm et al. (2007) proposed a

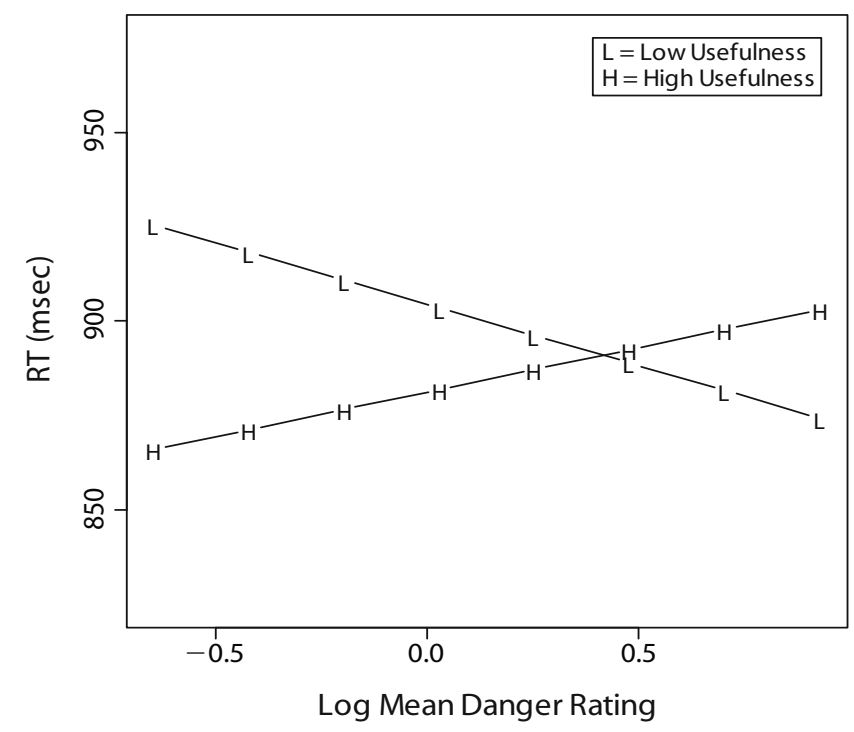

Figure 2. Results of the regression analysis predicting log RT as a function of danger and usefulness ratings. Neither danger nor usefulness was dichotomized for the analysis. Untransformed RTs are plotted. two-pass system in which early, fast, automatic evaluation (on one or a small number of dimensions) determines a general approach/avoid orientation, and semantics comes into play slightly later, helping to select a specific strategy.

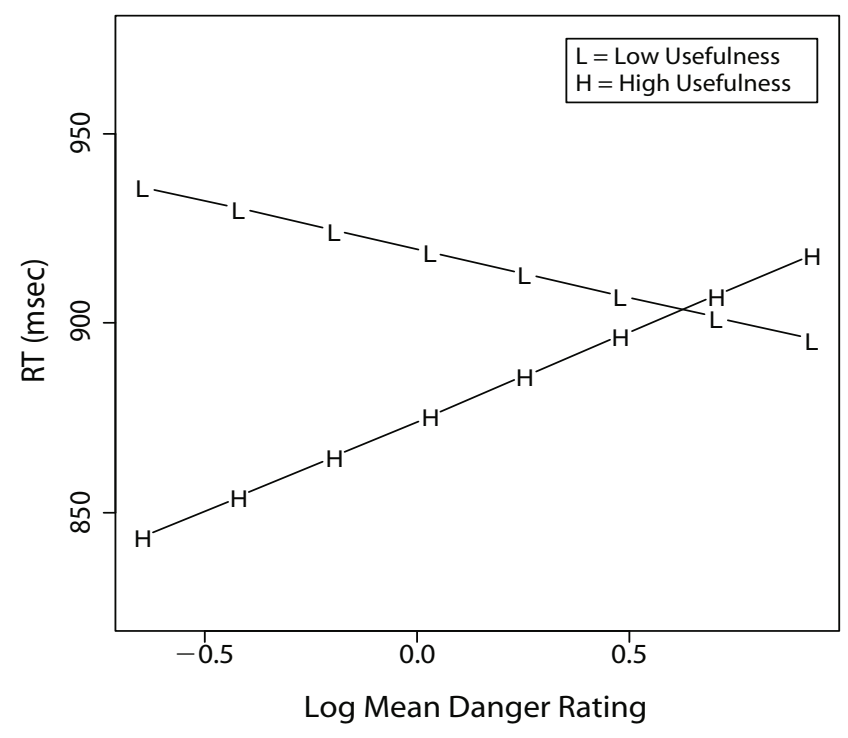

Figure 3. Results of a second regression analysis, analogous to that shown in Table 1, but with only frequency, duration, and UP location as control variables. Neither danger nor usefulness was dichotomized for the analysis. Untransformed RTs are plotted. 
In the complex situation of high usefulness combined with high danger, there will be more strategies that are adaptive in some way, which predicts the observed rise in RTs.

Neumann, Förster, and Strack (2003) point out the importance of using tasks that are not open to introspective processes when determining whether evaluation/behavior links extend to the level of representation. This is a major strength of the present study. It is extremely doubtful that any participant in the present study left the lab with any idea about the nature of the investigation (see Appendix A). The observed effects seem to reflect general, pervasive characteristics of perceptual processing. It should be emphasized that in the present study, these putative reflections of approach/avoid behavioral tendencies show themselves even when there is no reason why they should - participants simply had to decide whether a stimulus was a real word.

The present study extends the literature in several ways. First, it provides an alternative theoretical framework for understanding the widespread effects of evaluation/valence. There has been a return to the notion that such effects must relate to approach/avoid motivations (cf. Lewin, 1935; Schneirla, 1959); nevertheless, most modern discussions still emphasize evaluation/valence as the underlying dimension. As discussed above, though, there is difficulty in mapping good/bad onto approach/ avoid: $\mathrm{Bad}$ should sometimes be associated with approach behaviors, because bad things are sometimes useful. For example, syringe and spear are items from Wurm and Vakoch (2000) that ranked in the lowest quartile on evaluation but in the highest quartile on usefulness. Danger and usefulness offer an alternative conceptualization. Ours are the first studies to measure connotations of danger and usefulness directly, and relate them to online processing times in word recognition tasks.

Second, the present study has implications for models of cognitive/affective appraisal. Most demonstrations of evaluation/valence effects use some kind of priming manipulation, and words chosen because they are extreme on some evaluative dimension. There is nothing wrong with priming, and maximizing the differences between groups of stimuli is of course desirable. But effects in our studies emerge under circumstances taken as gauges of more ordinary perceptual processing. One need not select extreme items, and one need not prime the system.

A clever study by Moors and De Houwer (2001) showed that one can observe compatibility effects (i.e., between valence and behavioral response) with stimuli that are intrinsically neutral. However, their stimuli were made positive or negative through phony feedback in a computer game, and motivational valence was by definition important for part of the task. In addition, this study used a priming manipulation and item repetition, as have most studies on these topics.

There are at least two lexical decision studies that did not use priming, though. Neumann and Strack (2000) created an impression of "approach" or "avoid" by using shrinking or expanding concentric circles on a computer screen. Wentura, Rothermund, and Bak (2000) used two response types (the standard "Press button" plus a "With- draw finger" condition) in order to give the responses themselves an approach or avoid component. However, they also had to take additional steps to ensure that participants understood these as approach and withdraw reactions, either increasing or decreasing the size of the text when responses were made. Both studies used stimulus items extreme on valence.

These results are intriguing, but they also highlight the unique strengths of the present study. This is the first demonstration of such effects using the lexical decision task, with words not chosen for their evaluative extremity, with no repetition of items, with no primes preceding the targets, with no manipulation to ensure that subjects understood their responses in terms of approach/avoid, with no concurrent stimuli in the experiment to encourage any such understanding, and with no manipulation to impart any temporary valence to the stimuli. It is also the first study to examine such questions using auditory stimulus presentation. The effects are probably far more pervasive than researchers have thought.

This study has implications for models of word recognition, too. Because of the exhaustive nature of stimulus selection and statistical control of potential confounds, formal models will now have to address such effects. This is true whether danger/usefulness or some other framework is ultimately shown to be the most appropriate. No existing model is equipped to accommodate such effects.

Results of the present study argue for a perception/evaluation/action link, even for abstract symbols like words, of the type seen in embodied approaches (Barsalou, 2003; Glenberg, 1997; Margaret Wilson, 2002; cf. Lewin, 1935). Such a link has been demonstrated in a number of studies on the relation between evaluation/valence and motor responses. For example, Chen and Bargh (1999) found that participants were faster to pull than push a lever when categorizing positive stimuli, but that the reverse held for negative stimuli. There have been a number of extensions of this basic demonstration (e.g., De Houwer, Crombez, Baeyens, \& Hermans, 2001). In some, the effect is not one of evaluation influencing motor response, but motor response influencing evaluation. According to Neumann et al. (2003), “. . . the two behavioral orientations [approach/avoid] are not only a consequence, but also a cause of evaluative processes" (p. 374, emphasis mine). I believe the present effects are related to these pervasive evaluation effects because a reconceptualization of our own evaluation effects led to the present framework.

Some theorists have proposed separable positive and negative valence dimensions (e.g., Cacioppo, Gardner, \& Berntson, 1999), mapping positivity and negativity onto appetitive and aversive motivational systems. It could be argued on such a "bivariate valence" account that the observed slow-down in RTs is not due to an approach/avoid conflict, but to a mismatch between a stimulus's multiple valence values. The interpretational ambiguity discussed above reappears here, though: The difficulty in capturing what exactly good and bad mean is not alleviated by assigning each to its own dimension. In the proposed framework, we can say straightforwardly that the item is good because it is useful, and bad because it is dangerous. ${ }^{4}$ 
One way to contrast the proposed framework and the bivariate valence model of Cacioppo et al. (1999) would be to focus on stimuli that are both dangerous and useful (or alternatively, high on both positive and negative valence). My framework asserts that such items engage both approach and avoid behaviors. The bivariate valence model asserts that such stimuli elicit intense ambivalence (which could translate to longer RTs in lexical decision), but that they engage neither approach nor avoid behavior (Cacioppo et al., 1999, p. 843). Assuming a task can be used that taps into approach and avoid behaviors, it should be relatively easy to contrast the models.

Wurm and Seaman (in press) report that danger and usefulness explain 5.5\% more variance in by-item auditory naming times for common nouns, in comparison with Osgood's evaluation/potency/activity framework. I was also able to perform post hoc analyses for the 55 items in the present study that are listed in the Bradley and Lang (1999) valence and arousal norms. These were similarly encouraging. First, danger and usefulness explain 3\% more variance in by-item RTs than do valence and arousal. Second, the variable that correlates most strongly with mean RT is usefulness (valence is second best). Third, in a regression analysis with valence, arousal, danger, and usefulness as predictors, only usefulness is a significant predictor of RT (danger and valence are marginal; arousal is n.s.). Finally, the danger $\times$ usefulness interaction remains highly significant when valence and arousal are added to the analysis shown in Table $1(p=.0038)$. It is worth noting that valence and arousal are given access to the variance prior to the interaction term in such an analysis.

Thus, the framework has much to recommend it. It explains at least as much variance as do more established frameworks, and it seems to suffer from less interpretational ambiguity. The proposed framework is based on principles related to adaptiveness and the function of action, and it allows for testable predictions to be made in future research.

Accounts relating valence to behavioral dispositions generally stipulate that the temporary motivational state, specific to a particular organism at a particular time, will determine the nature of the approach/avoid behavior (Lewin, 1935; Schneirla, 1959). This might be true for items relating clearly to the specific motivational state (e.g., food items for someone on a diet; career-oriented items for someone seeking a job; see Brendl \& Higgins, 1996; Ferguson \& Bargh, 2004). It will be interesting to see to what extent these temporary, specific motivational dispositions can override the more permanent, general goal of survival.

Future research should also address the rating task itself. Most objects are dangerous or useful to varying degrees in different situations, so it is not clear what exactly participants base their ratings on: It could be the first thing that comes to mind, or a maximum value, or an average, or something else. The task seems difficult, and yet there is good within-item agreement on the ratings $(M S E M=0.22$ for danger and 0.26 for usefulness in the present study).

It is conceivable that wording the rating question differently (e.g., specifically mentioning survival of the species vs. of the rater - cf. Wentura et al., 2000; or anchoring the ratings not with respect to survival but to career advancement) would produce different ratings. The theory I am proposing predicts that the former manipulation would not make much difference and that the latter might; but danger and usefulness (scaled with respect to career goals) should not be predictive of lexical decision times. These are empirical questions. For the present, it is noteworthy that whatever participants are doing when they give danger and usefulness ratings, these ratings are related to RTs in a predictable way across tasks and item sets.

\section{AUTHOR NOTE}

I am indebted to Harald Baayen and Robert Schreuder for being such kind and generous hosts during a sabbatical leave. I also thank Matt Ventimiglia for recording stimuli, and Lynne Daigle, Lisa Van Havermaet, and Sean Seaman for help collecting data. Correspondence concerning this article should be addressed to L. Wurm, Department of Psychology and Program in Linguistics, Wayne State University, 5057 Woodward Avenue, 7th Floor, Detroit, MI 48202 (e-mail: lee.wurm@wayne.edu).

\section{REFERENCES}

Batyen, R. H., Feldman, L. B., \& Schreuder, R. (2006). Morphological influences on the recognition of monosyllabic monomorphemic words. Journal of Memory \& Language, 55, 290-313.

BaAyen, R. H., Piepenbrock, R., \& Gullikers, L. (1995). The CELEX lexical database [CD-ROM]. Linguistic Data Consortium. Philadelphia: University of Pennsylvania.

BaAyen, R. H., Wurm, L. H., \& Aycock, J. (in press). Lexical dynamics for low-frequency complex words: A regression study across tasks and modalities. The mental lexicon.

Balota, D. A., Cortese, M. J., Hutchison, K. A., Neely, J. H., Nelson, D., Simpson, G. B., \& Treiman, R. (2002). The English Lexicon Project: A web-based repository of descriptive and behavioral measures for 40,481 English words and nonwords. http://elexicon.wustl .edu/, Washington University.

BARSALOU, L. W. (2003). Situated simulation in the human conceptual system. Language \& Cognitive Processes, 18, 513-562.

Bradley, M. M., \& LANG, P. J. (1999). Affective norms for English words (ANEW): Stimuli, instruction manual and affective ratings. Technical report C-1, Gainesville: University of Florida, Center for Research in Psychophysiology.

Brendl, C. M., \& Higgins, E. T. (1996). Principles of judging valence: What makes events positive or negative? In M. P. Zanna (Ed.), Advances in experimental social psychology (Vol. 28, pp. 95-160). San Diego: Academic Press.

Burnard, L. (1995). Users guide for the British National Corpus. British National Corpus Consortium, Oxford University Computing Service.

Cacioppo, J. T., Gardner, W. L., \& Berntson, G. G. (1999). The affect system has parallel and integrative processing components: Form follows function. Journal of Personality \& Social Psychology, 76, 839-855.

Chen, S., \& BARgh, J. A. (1999). Consequences of automatic evaluation: Immediate behavior predispositions to approach or avoid the stimulus. Personality \& Social Psychology Bulletin, 25, 215-224.

De Houwer, J., Crombez, G., Baeyens, F., \& Hermans, D. (2001). On the generality of the affective Simon effect. Cognition \& Emotion, 15, 189-206.

Ellis, A. W., \& Morrison, C. M. (1998). Real age-of-acquisition effects in lexical retrieval. Journal of Experimental Psychology: Learning, Memory, \& Cognition, 24, 515-523.

Ferguson, M. J., \& BARGH, J. A. (2004). Liking is for doing: The effects of goal pursuit on automatic evaluation. Journal of Personality \& Social Psychology, 87, 557-572.

Glenberg, A. M. (1997). What memory is for. Behavioral \& Brain Sciences, 20, 1-55.

LEWIN, K. (1935). A dynamic theory of personality: Selected papers. New York: McGraw-Hill.

Miller, G. A. (1990). WordNet: An on-line lexical database. International Journal of Lexicography, 3, 235-312.

Moors, A., \& De Houwer, J. (2001). Automatic appraisal of moti- 
vational valence: Motivational affective priming and Simon effects. Cognition \& Emotion, 15, 749-766.

Neumann, R., Förster, J., \& Strack, F. (2003). Motor compatibility: The bidirectional link between behavior and evaluation. In J. Musch \& K. C. Klauer (Eds.), The psychology of evaluation: Affective processes in cognition and emotion (pp. 371-391). Mahwah, NJ: Erlbaum.

Neumann, R., \& Strack, F. (2000). Approach and avoidance: The influence of proprioceptive and exteroceptive cues on encoding of affective information. Journal of Personality \& Social Psychology, 79, 39-48.

OsGood, C. E. (1969). On the whys and wherefores of E, P, and A. Journal of Personality \& Social Psychology, 12, 194-199.

Pinheiro, J. C., \& Bates, D. M. (2000). Mixed-effects models in S and $S$-PLUS. New York: Springer.

Robinson, M. D., Storbeck, J., Meier, B. P., \& Kirkeby, B. S. (2004). Watch out! That could be dangerous: Valence-arousal interactions in evaluative processing. Personality \& Social Psychology Bulletin, 30, 1472-1484.

SchneIRLA, T. C. (1959). An evolutionary and developmental theory of biphasic processes underlying approach and withdrawal. In M. Jones (Ed.), Nebraska Symposium on Motivation (pp. 1-42). Lincoln: University of Nebraska Press.

Schreuder, R., \& BAAYEN, R. H. (1997). How complex simplex words can be. Journal of Memory \& Language, 37, 118-139.

Solarz, A. K. (1960). Latency of instrumental responses as a function of compatibility with the meaning of eliciting verbal signs. Journal of Experimental Psychology, 59, 239-245.

Wentura, D., Rothermund, K., \& BAK, P. (2000). Automatic vigilance: The attention-grabbing power of approach and avoidance-related social information. Journal of Personality \& Social Psychology, 78, 1024-1037.

Wilson, Margaret (2002). Six views of embodied cognition. Psychonomic Bulletin \& Review, 9, 625-636.

Wilson, Michael (1988). MRC psycholinguistic database: Machineusable dictionary, Version 2.00. Behavior Research Methods, Instruments, \& Computers, 20, 6-10.

Wurm, L. H., Ernestus, M., Schreuder, R., \& BaAyen, R. H. (2006). Dynamics of the auditory comprehension of prefixed words: Cohort entropies and conditional root uniqueness points. The Mental Lexicon, 1, 125-146.

Wurm, L. H., Labouvie-Vief, G., Aycock, J., Rebucal, K.A., \& Косн, H. E. (2004). Performance in auditory and visual emotional Stroop tasks: A comparison of older and younger adults. Psychology \& Aging, 19, 523-535.

Wurm, L. H., \& SeAman, S. R. (in press). Semantic effects in naming and perceptual identification, but not in delayed naming: Implications for models and tasks. Journal of Experimental Psychology: Learning, Memory, \& Cognition.

WURM, L. H., \& VAKOCH, D. A. (2000). The adaptive value of lexical connotation in speech perception. Cognition \& Emotion, 14, 177-191.

Wurm, L. H., Vakoch, D. A., Aycock, J., \& Childers, R. R. (2003). Semantic effects in lexical access: Evidence from single-word naming. Cognition \& Emotion, 17, 547-565.

Wurm, L. H., VaKoch, D. A., \& Seaman, S. R. (2004). Recognition of spoken words: Semantic effects in lexical access. Language \& Speech, 47, 175-204.

Wurm, L. H., Vakoch, D. A., Seaman, S. R., \& Buchanan, L. (2004). Semantic effects in auditory word recognition. Mental Lexicon Working Papers, 1, 47-62.

Wurm, L. H., Whitman, R. D., Seaman, S. R., Hill, L., \& UlSTAD, H. M. (2007). Semantic processing in auditory lexical decision: Ear-of-presentation and sex differences. Cognition \& Emotion, 14, 1470-1495.

Zevin, J., \& Seidenberg, M. (2002). Age of acquisition effect in word reading and other tasks. Journal of Memory \& Language, 47, 1-29.

\section{NOTES}

1. I intend the terms approach and avoid to mean a tendency of the observer to reduce or increase the distance to a location/object/event (Solarz, 1960). Also, I use the familiar term avoid rather than withdraw, which Schneirla (1959) points out would be more technically correct.

2 . These constraints were used for purposes of simplification. Including morphologically complex words, for example, would create a need for an additional constellation of control variables; and whereas I do predict that danger and usefulness effects would be found for such words, the question will have to await further research. Using the ELP as the source for stimuli also allows for researchers interested in visual processing to have easy access to the wide range of orthographic information about each stimulus that is available there.

3. I reran the subanalysis on nouns, substituting the actual AoA values from the MRC database for the surrogate "written-to-spoken ratio." This had no effect on the interaction $[B=0.0596, S E B=0.0253, t(5427)=$ $2.3534, p=.0186]$.

4. The danger/usefulness framework seems plausible as the basis of a causal model, too. It seems possible for an item to get a positive valence rating because it is useful, but less possible for an item to get a high usefulness rating because it is positively valenced. Similarly, I believe an item can get a negative valence rating because it is dangerous; the reverse seems less likely.

\section{APPENDIX A}

\section{Mean Danger and Usefulness Ratings}

Nouns. apple $(1.56,5.87)$, area $(2.14,4.65)$, ash $(2.51,2.69)$, basin $(1.94,3.8)$, bird $(2,4.79)$, blade $(5.29$, $5.11)$, booth $(1.61,2.57)$, brass $(1.96,3.61)$, breath $(2.85,7.32)$, brick $(2.99,4.79)$, canteen $(1.93,4.94)$, car (3.61, 5.28), chicken $(2.11,6.11)$, coffee $(2.65,3.76)$, crime $(6.29,3.51)$, deceit $(4.94,4.03)$, diamond $(2.4,3.08)$, doll $(1.6,2.75)$, door $(1.72,4.36)$, elephant $(2.81,3.99)$, elm $(1.71,3.48)$, forest $(2.92,5.65)$, fraud $(5.18,3.52)$, frog $(1.82,3.83)$, germ $(5.93,5.01)$, girl $(2.56,6.47)$, globe $(2.38,4.89)$, glove $(1.71,4.42)$, grape $(1.31,5.49)$, heart $(3.57,7.28)$, hero $(2.21,5.18)$, herring $(1.8,4.22)$, honey $(1.85,4.61)$, horn $(2.47,3.32)$, hospital (3.72, 6.96), hotel $(1.87,4.04)$, insect $(3.19,4.38)$, juice $(1.64,5.79)$, kettle $(2.04,4)$, kitten $(1.65,3.33)$, lad $(1.97,3.9)$, lady $(2.28,6.29)$, lamp $(1.71,4.28)$, law $(3.61,6.19)$, lemon $(1.78,4.69)$, lily $(1.31,3.35)$, linen $(1.64,3.99)$, lion $(4.75,4.31)$, loss $(4.45,4.08)$, magazine $(1.76,2.78)$, method $(2.57,5.58)$, mile $(1.67,3.67)$, minnow $(1.57$, $3.63)$, moment $(2.24,4.54)$, money $(5.1,6.07)$, mountain $(3.47,4.47)$, movie $(1.79,3.47)$, mud $(1.99,3.51)$, mule $(2.34,4.65)$, music $(1.96,5.12)$, napkin $(1.36,3.19)$, noun $(1.66,4.4)$, oak $(1.56,4.49)$, ocean $(3.64,5.78)$, organ $(3.26,6.32)$, oven $(3.19,5)$, owl $(1.79,3.69)$, palace $(2.07,3.46)$, peach $(1.47,5.65)$, pigeon $(1.85,3.72)$, potato $(1.67,5.82)$, priest $(2.54,4.06)$, problem $(4.75,4.87)$, rug $(1.53,3.08)$, sardine $(1.74,4.24)$, sequel $(2$, 2.94), shawl $(1.7,3.53)$, sleeve $(1.43,3.6)$, song $(1.42,4.67)$, steeple $(2.03,3.16)$, stool $(2.14,3.33)$, stove $(3.18$, $5.1)$, straw $(1.67,3.87)$, sword $(5.19,4.85)$, theft $(5.5,3.67)$, thief $(5.9,3.32)$, threat $(6.33,4.35)$, tobacco $(5.87$, $2.28)$, tomb $(3.58,3.27)$, ton $(2.58,3.37)$, trance $(2.83,2.85)$, trout $(1.99,5.11)$, tunic $(2.18,3.39)$, umbrella $(1.72,3.87)$, uncle $(1.87,4.81)$, vase $(1.64,2.71)$, verb $(1.74,4.67)$, village $(2.19,5.57)$, wheat $(1.86,6)$, window $(2.15,4.74)$, woman $(2.97,7.03)$, yard $(1.68,3.69)$, year $(2.11,4.65)$, youth $(2.47,5.97)$ 


\section{APPENDIXA (Continued)}

Verbs. adorn $(1.77,3.41)$, allow $(2.42,5.31)$, appear $(2.04,4.22)$, assist $(2.29,5.58)$, beg $(3.06,3.49)$, condemn $(4.46,4.07)$, fail $(4.74,4.36)$, forbid $(3.96,4.81)$, get $(2.22,5.14)$, give $(2.26,5.6)$, grow $(2.62,6.72)$, ignore $(3.63,3.74)$, keep $(2.29,4.82)$, kindle $(2.2,3.91)$, learn $(2.47,7.11)$, lose $(3.89,4.03)$, melt $(2.81,4.39)$, prohibit $(3.81,5)$, prosper $(2.17,5.86)$, put $(1.79,3.82)$, retain $(2.46,5.22)$, send $(2,4.22)$, sever $(5.24,4.09)$, sing $(1.4,4.26)$, sit $(1.94,4.87)$, speak $(2.83,6.85)$, stifle $(3.05,3.19)$, succeed $(2.6,6.69)$, take $(3.33,4.53)$, teach $(2.46,6.88)$, tell $(2.47,5.24)$, think $(2.89,7.4)$, want $(3.6,5.01)$, weep $(2.85,4.14)$

Adjectives. afraid $(4.69,4.56)$, apt $(1.91,3.48)$, astute $(1.89,4.17)$, avid $(2.06,3.28)$, bad $(4.92,4.04)$, bland $(1.84,2.9)$, bleak $(2.81,3.02)$, bright $(2.28,4.99)$, clever $(2.46,5.66)$, drab $(2.47,2.89)$, finite $(2.8,4.62)$, frail $(4.1,3.6)$, frigid $(3.87,3.64)$, gentle $(1.81,5.18)$, good $(1.81,6.17)$, humane $(2.71,6.2)$, lax $(2.07,3.25)$, mad $(4.42,4.54)$, meek $(2.12,3.37)$, mild $(2.11,3.61)$, neat $(1.58,4.46)$, nice $(1.87,5.43)$, obese $(6.18,2.93)$, pious $(2.39,3.08)$, polite $(1.82,5.32)$, rare $(2.79,4.01)$, ripe $(1.89,4.97)$, sad $(3.79,4.28)$, scarce $(4.85,4.37)$, severe $(5.4,4.14)$, simple $(1.92,4.74)$, soft $(1.56,4.29)$, solemn $(2.07,3.69)$, tall $(2.04,4.1)$, tranquil $(2.62,4.62)$, vague $(2.94,3.13)$, weird $(2.69,3.5)$

Note-The first number in parentheses is the mean danger rating. The second is the mean usefulness rating. Danger and usefulness values were log-transformed and centered prior to statistical analyses.

\section{APPENDIX B}

Control Variables

1. Whether or not there was an error on the previous trial. Participants often slow down after an error, probably a surprise or realerting effect.

2. Trial number, which controls for variance due to practice or fatigue effects.

3. First-syllable stress.

4. Voicing of the initial phoneme. Baayen, Wurm, and Aycock (in press) found that voiced onsets predict faster auditory RTs.

5. Acoustic duration, and the point at which each item becomes unique (i.e., the auditory uniqueness point, or UP)

6. Coltheart's $N$ is the number of words that can be made from a target word by the substitution of one letter. Number of competitors is the auditory cohort size one phoneme prior to the UP.

7. Summed bigram frequency values, from the English Lexicon Project (Balota et al., 2002).

8. Surface frequency values were taken from the CELEX database (Baayen et al., 1995).

9. Number of meanings. I used the number of synonym sets each word has in WordNet (Miller, 1990). A synonym set is a group of semantically related words.

10. Morphological family size is the number of compounds and derived words in which a given word appears as a constituent (e.g., Schreuder \& Baayen, 1997). It has been found to predict faster auditory lexical decision times (Wurm, Ernestus, Schreuder, \& Baayen, 2006).

11. Concreteness, imageability, familiarity, and meaningfulness were taken from the MRC database. Concreteness and imageability correlated $.928(p<.0001)$, so imageability was not used.

12. Age of acquisition (for nouns) was taken from the MRC database. Very few nonnouns have an AoA value in the MRC database. I therefore used the ratio of written to spoken frequencies from the British National Corpus (Burnard, 1995). A higher written-to-spoken ratio indicates a word that is likely to have been learned later (Baayen, Feldman, \& Schreuder, 2006), so we expect a positive correlation with AoA. For the nouns, which have AoA values, this can be calculated $[r(102)=.602, p<.0001]$. 